\title{
«Agir par son être-même»: La question de la liberté créatrice dans le Liber de causis et sa réception chez Albert le Grand
}

\author{
Isabelle Moulin \\ Université de Strasbourg
}

Dans le contexte d'une production du monde définie en termes de création, l'exigence d'une liberté du premier principe ne va pas de soi. En effet, une fois éliminée son antithèse qui se définirait comme la domination toute-puissante du maître sur l' esclave et qui est indigne d' un homme libre, dans toute la philosophie antique, la nécessité est précisément la marque d'un plus grand degré d'être, d' une plus grande noblesse ontologique et d'une continuité dans l'action, indice premier de la sagesse. Pour Aristote, seuls les êtres du monde supra-lunaire agissent de manière nécessaire, continue et uniforme, tandis que le monde sublunaire n'est que le lieu de ce qui arrive «le plus souvent» ou «rarement». Ces éléments sont encore plus évidents dans le cas du premier principe qui est un être éminemment nécessaire et dont la nature est le Bien. Il n' a pas à choisir entre le bien et le mal puisqu' il ne peut agir que conformément à son être. Dans ces conditions, quelle serait une liberté de production qui résulterait d'un choix d'agir ou de ne pas agir, voire même de produire un autre monde? En particulier, l'émanation des êtres à partir du premier principe ne se produit pas sous l' effet du hasard et suit un ordre nécessaire. C'est même cette nécessité qui a conduit à son rejet comme modèle de la création en théologie.

Notre étude vise à interroger la question de la nécessité de l'émanation et à concevoir, à la suite d'Albert le Grand, une possibilité d'intégration de ce modèle dans le contexte plus global d' une réflexion sur la création. Or le Liber de causis est l' un des textes fondateurs de cette association. Il sera donc notre point de départ. En proposant une lecture du traité 39, très controversé, des Ennéades de Plotin, nous montrerons ensuite que les conclusions du Liber se rapprochent davantage du milieu plotinien que proclusien créant ainsi une sorte d'unité interprétative dans la tradition latine. Enfin, nous verrons comment Albert le Grand transforme cet héritage en l'inscrivant dans une conception particulière de l'être divin. 
Le Liber de causis ${ }^{1}$ propose une voie propre de «reprise » de la tradition plotinoproclusienne dans le contexte philosophique de la création, envisagée dans son activité dynamique de diffusion du Bien. Il ne s' offre donc pas seulement comme un complément de la Métaphysique d'Aristote. S'inscrivant dans la lignée d'un nouveau type de «causalité» qui marque l' insuffisance fondamentale des quatre causes aristotéliciennes pour désigner l'acte créateur, il prend sa place dans le monde latin au XıI I ${ }^{\text {ème }}$ siècle, comme référence philosophique incontournable de toute réflexion sur la création. Ainsi, lorsqu' ils traitent de cette question, Albert le Grand et Thomas d' Aquin font systématiquement référence au Liber de causis, y compris dans leurs ouvrages théologiques, comme les Sommes ou les Commentaires des Sentences, relativisant, sans le nier, le poids de l' autorité théologique d' Augustin².

Sur la question propre de l'acte créateur ${ }^{3}$, qu' apporte le Liber par rapport au fonds commun du néoplatonisme grec et en particulier à Proclus dont il est tiré? Tout d' abord il redéfinit le monisme proclusien en un monothéisme par un processus d' unification et d' articulation de la diversité des théophanies proclusiennes de l'Un; puis il introduit la notion de création qui implique la mise en place d' une nouvelle articulation entre le Créateur et les créatures par une série de distinctions binaires, fondées sur un certain nombre de critères qu' il ne réussit pas toujours à unifier ${ }^{4}$. Cette binarité redéfinit les contours de la

1 Je cite d'après Magnard, Boulnois, Pinchard, Solère 1990.

2 Pour autant, le rapport des deux autorités du Liber et d'Augustin ne se posent pas en termes antagonistes chez ces auteurs, et notamment chez Albert, puisque l'on assiste à un subtil travail d'harmonisation favorisé par le platonisme d'Augustin et s'appuyant notamment sur deux thèses fondamentales communes à Plotin et à Augustin: les raisons séminales et l'intellection comme processus d' illumination. Voir Klibansky 1937, p. 23.

3 Voir Burrell 1993 et 2003 .

4 Citons l'antécédence au concept d'éternité (Liber de causis II, 2O-21, p. 40-41: «l'être qui est avant l'éternité est la cause première, puisqu'il en est la cause. Mais l'être qui est avec l'éternité est l'intelligence»); l'antécédence au concept d'infinité (Liber de causis Xv, ${ }_{136}$, p. 64-65: «l'être premier qui est créateur est au-dessus de l'infini, mais [...] l'être second qui est créé est infini; et ce qui se trouve entre l'être premier créateur et l'être second créé est le non-fini»); la distinction du per modum creationis et du per modum formae (Liber de causis XVII, 148, p. 66-67); et plus globalement l'ordre émanatif de l'être et de l'intelligence comme premiers créés. Si Albert le Grand reprend la plupart de ces éléments, c'est pour les unifier dans le rapport boécien de l'id quod est et du quod est ou encore de la distinction de l'essence et de l'existence avicénienne; mais également pour gommer l'excès de transcendance qu'implique l'au-delà de l'être, de l'éternité et de l'infinité. Autrement dit, l'étape supplémentaire albertinienne consistera à introduire une relation entre les deux pôles dégagés par le Liber. 
transcendance du premier principe et celle de sa liberté. Ainsi, alors que les Éléments de théologie de Proclus, «betrays no preoccupation with Christianity», comme le souligne E.R. Dodds ${ }^{5}$, et que Proclus «semble ignorer splendidement les chrétiens et la doctrine chrétienne», comme le remarque H.D. Saffrey, le Liber de causis permet une «christianisation» de la pensée de Proclus, selon une ironie historique dont la translatio studiorum a le secret. Enfin, il rencontre l'objection de la nécessité de l'émanation, par opposition à un acte créateur libre.

Il est vrai qu'à première vue, le Liber ne semble pas remettre en cause le schéma interprétatif de l'émanation nécessaire. En effet, l' influx originaire se produit de manière aveugle et suit un déroulement causal totalement prédéterminé selon l' ordre logique de la structuration porphyrienne du plus général au plus spécifique. C' est l'un des sens possibles de la célèbre formule: omnis intelligentia plena est formis ${ }^{7}$. De même, le rapport du premier créé aux formes qu'il crée est pensé sous la forme de l' espèce et des individus ${ }^{8}$. La forme-même du traité divisé en éléments logiques et adoptant une structure déductive renforce cette impression. De plus, la nécessité du Premier semble se transmettre dans la chaîne causale qui efflue de lui. Enfin, l'élément de séparabilité est déterminant pour une conception de la liberté créatrice: trop transcendante, la cause première donne le premier influx nécessaire et l'ensemble du créé se déroule selon l'ordre préétabli des intermédiaires; trop immanente, l' action de la première cause devient co-extensive à ce qu' elle crée et ne laisse place à aucune initiative libre des causes secondes. Or, la thèse de la création mediante intelligentia n' est pas facile à évaluer. Doit-elle s' entendre comme l' expression d'un vrai intermédiaire, comme c' est le cas dans l'émanation avicennienne ou bien comme une causalité seconde ou instrumentale qui préserve l' ensemble de l' acte du premier principe ${ }^{9}$ ? Pour donner une bonne appréciation de ce problème du déterminisme causal de l'émanation dans le Liber, il faut se reporter à deux moments principaux qui posent la liberté créatrice: le chapitre XXII et le chapitre XIX.

Le chapitre XXII du Liber de causis étudie globalement le rapport de la cause première aux choses créées du point de vue du «gouvernement», terme qui

5 Dodds 1963, p. 188.

6 Saffrey 1990, p. 202 (554).

7 Liber de causis IX, 92, p. 56-57.

8 Liber de causis IV, 45, p. 44-45: «De même que, d'une forme unique, du fait qu' elle est diversifiée dans le monde inférieur, proviennent des individus infinis en nombre, de même de l'être premier créé, du fait qu' il est diversifié, surgissent des formes intelligibles à l' infini ».

9 Voir D’Ancona 1995, en particulier p. 73-75. 
désigne à la fois l'extension de la capacité causale et l'action providentielle. Ces deux éléments ne doivent pas être simplement juxtaposés: le gouvernement marque la continuité d' une action causale et exprime fondamentalement la notion de donation. Le causé n'est plus seulement le résultat ou le produit d' une action causale, un simple donné, mais il est l' objet d'une action continue et d'une relation permanente; à l'opposé d'une conception purement mécaniste de la causalitét ${ }^{0}$.

Ce chapitre sur le gouvernement divin s' inscrit de manière très spécifique dans le contexte global de l' ouvrage. C' est en effet le seul moment où la mention de «Dieu» apparaît à plusieurs reprises, assortie de qualifications nettement religieuses (benedictus, sublimis, excelsus), où le terme-même de création est explicitement appliqué à la cause première qui prend le nom de «Créateur de l'intelligence»; mais c'est également la seule occurrence qui attribue la «volonté» au premier principe, assurant la liberté de l'acte créateur:

172. Omnis intelligentia divina scit res per hoc quod ipsa est intelligentia, et regit eas per hoc quod est divina.

173. Quod est qui proprietas intelligentiae est scientia, et non est eius complementum et integritas nisi ut sit sciens. Regens ergo est Deus, benedictus et sublimis, quoniam ipse replet res bonitatibus. Et intelligentia est primum creatum et est plus similis Deo sublimi, et propter illud regit res quae sub ea sunt. Et sicut Deus benedictus et excelsus influit bonitatem super res, similiter intelligentia influit scientiam super res quae sunt sub ea.

174. Verumtamen, quamvis intelligentia regat res quae sunt sub ea, tamen Deus benedictus et sublimis praecedit intelligentiam per regimen et regit res regimine sublimioris et altioris ordinis quam sit regimen intelligentiae, quoniam est illud quod dat intelligentiae regimen.

175. Et significatio illius est quod res, quae recipient regimen intelligentiae, recipient regimen creatoris intelligentiae, quod est quia non refugit

Il ne signifie plus seulement l' opération rectiligne de l' action descendante de la cause originant le causé, mais un acte que l' on peut comparer au mouvement hélicoïdal dionysien

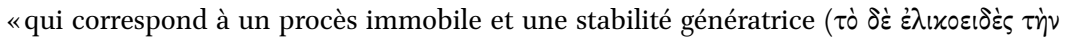

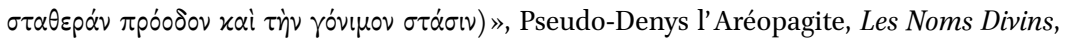
IX, 916C-D, trad. de Gandillac p. 16o. Dieu est immobile mais son action s' exprime selon trois «mouvements » : le mouvement circulaire qui «signifie que Dieu demeure identique à soi-même», le mouvement en ligne droite qui «doit s'entendre comme le procès sans détours ni déclinaison des opérations divines, comme la naissance de toutes choses à partir de Dieu même» et le mouvement hélicoïdal qui «correspond à un procès immobile et une stabilité génératrice». 
regimen eius aliqua ex rebus omnino, quoniam vult ut faciat bonitatem suam simul omnes res. Quod est quia non est quod omnis res desiderat intelligentiam nec desiderat recipere eam, res omnes desiderant bonitatem ex primo et desiderant recipere ipsam desiderio multo. In illo non est aliquis qui dubitet ${ }^{11}$.

Sur la question de la transcendance, on voit que la causalité des êtres seconds, et en particulier du premier créé, ne se substitue en rien à celle du Créateur $^{12}$. L' ordre du gouvernement préserve l'action première de Dieu car si toutes choses ne peuvent recevoir le gouvernement du premier créé, qui est l'intelligence, toutes, en revanche désirent le Premier. La séparabilité est également bien soulignée en ce que la cause première n' est en rien mélangée aux choses créées: «la cause première régit toutes les choses créées, sans qu'elle soit mêlée avec elles » ${ }^{13}$. La distinction du per modum creationis et du per modum formae, attribuant le seul acte créateur au Premier, témoigne de cette séparabilité. Enfin, l'usage propre du Liber qui définit l'être-dans selon une modalité que nous pourrions appeler la règle d'inhérence formelle dissocie ontologiquement la cause de l'effet selon le mode propre à chacun. La première occurrence apparait au $\S 75$ et trouve sa formulation au § 106: «L' effet est dans la cause selon le mode de la cause, et la cause est dans l'effet selon le mode de l' effet » ${ }^{14}$. A l'ensemble de ces éléments, l'adjonction discrète du hapax sur la volonté du Premier modifie le caractère problématique de la distinction du creare et de l' informare: que les choses reçoivent le gouvernement de l'intelligence ou non, toutes entrent en relation avec le Créateur en tant qu' elles sont voulues par lui et que toutes le désirent. La mention de la volonté est un effet propre du caractère primordial du Bien et de l' amour sur celui de l'intelligence. Étant plus universel, il atteint toutes créatures, même celles qui sont dénuées de rationalité.

La question de la relation non-réciproque et celle de la dépendance et du retour du créé dans le Créateur, qui sont des éléments nécessaires pour définir toute liberté créatrice, semblent donc relativement bien honorées par l' auteur anonyme du Liber ${ }^{15}$. En revanche, au chapitre XII, mais surtout aux chapitres

\footnotetext{
11 Liber de causis XXII, 172-175; c' est nous qui soulignons.

12 Le Liber s' ouvre sur cette sentence: Omnis causa primaria plus est influens super causatum suum quam causa universalis secunda (Liber de causis I, 1, p. 38-39). Voir également Liber de causis VII, 86, p. 54-55.

13 Liber de causis XIX, 155, p. 68-69.

14 Liber de causis XI, 106, p. 58-59.

15 Certaines formulations semblent encore problématiques ou, du moins, nécessiteront
} 
XIX et XX, il utilise une formulation qui est éminemment problématique. Le Premier n' use pas d'intermédiaire dans son acte créateur, parce qu' il ne saurait exister d'intermédiaire entre son être et son faire: «il gouverne par le mode sur lequel il agit et il n'agit que par ce qu'il est, donc son être est également son gouvernement ${ }^{16}$. Si agir par son être se définit comme une nécessité de nature, peut-on dire que le Premier est libre de créer lorsqu'il est contraint à se diffuser? L'acte créateur ne peut se définir comme libre si la nature du Premier, qui est celle du Bien diffusif de soi, rend nécessaire l' acte créateur.

Le rapport du faire à l' être du chapitre XIX du Liber se présente bien comme un enjeu fondamental pour la question de la liberté de l'acte créateur mais le Liber ne livre que très peu d'éléments sur la manière dont il faut concevoir une telle identité qui semble remettre en cause toute liberté originaire du premier principe. Comme l'a si bien montré Jean Trouillard à propos de Proclus, qui est l'une des sources du Liber ici, la formulation «Agir par son être-même »17 interroge d'emblée la question de la liberté18.

Le Liber pose ainsi un certain nombre de jalons fondamentaux pour une réflexion sur le sujet de la création. Par ailleurs, il répond bien à la problématique du rapport de l'être au faire, mais sans en relever les conséquences pour la question de la liberté. Il convient alors de se tourner vers un autre texte fondateur sur la question : le célèbre traité 39 de Plotin ${ }^{19}$.

d'être mieux élucidées: l'interprétation du mediante intelligentia et surtout la question de la pré-contenance des formes dans les principes intelligibles; le principe de la diversité réceptrice ne permet pas de résoudre systématiquement les questions de différenciation.

16 Liber de causis, XX, 161, p. 70-71; voir également: Liber de causis XIX, 159, p. 70-71: «entre tout agent qui agit par son seul être, et ce qu'il fait, il n'y a pas de moyen-terme s' entremettant ni d' autre chose intermédiaire» et XIX, 160, ibid.: «Mais l' agent qui n' admet absolument pas d'intermédiaire entre lui-même et ce qu'il fait est un agent et gouvernant véritable». Comme l' a montré A. Pattin, la notion du mediante intelligentia semble émaner de la distinction entre la cause démiurgique et la cause paternelle de la proposition 157 de l'Elementatio. Mais le medium ne s' entend pas encore comme cause instrumentale. Voir Pattin 1994, aux pages 368-370 (208-210).

17 Proclus, In Timaeum, p. 393.

18 Trouillard 1958. Il s' agit de la formulation proclusienne des «véritables puissances»: $A i$

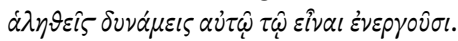

19 Nous ne cherchons pas à construire une chaîne des sources, mais seulement à montrer comment certains textes fondateurs ont donné lieu à un faisceau d'arguments qui ont permis de construire et de répondre à une problématique; ici, celle de la liberté du premier principe. Le traité 39 de Plotin est fondateur car la question de la volonté du premier principe n' a que peu ou pas du tout été interrogée chez ses prédécesseurs, comme le souligne Leroux 1990: «De manière radicale, la proposition de la volonté divine comme volonté de soi apparaît comme une proposition originaire chez Plotin. Elle est sans antécédents 
Au livre VI, chapitre 8 des Ennéades (traité 39), Plotin traite de «la liberté et la volonté de l'un $»^{20}$. Dans le cadre de la liberté humaine, Plotin aboutit à une identité entre la volonté et la pensée lorsque la volonté atteint son objet qu' est le Bien ${ }^{21}$, et c' est dans l'identité que se situent la liberté et l' autonomie humaines. Qu' en est-il du Bien? La question de la liberté du Bien est soulevée à la suite d' une objection «audacieuse», qui pose le hasard à l' origine du premier principe. Un tel «discours téméraire» ouvre, en le niant, le questionnement de la liberté de l' Un et ne laisse d' autre possibilité que la nécessité de nature, thèse qui est celle d'Alexandre d'Aphrodise qui semble être le vrai interlocuteur de Plotin dans ce texte ${ }^{22}$.

La réponse de Plotin est particulièrement novatrice. Elle tient dans l' absurdité d' une position qui sera celle du pur libre-arbitre ou de la liberté d'indifférence de Descartes: « dire qu'il n' est pas libre parce qu'il produit selon ce qu' il est, c' est absurde; c' est juger qu' être libre, c' est produire ou agir contrairement

et on ne peut la ramener à aucune doctrine philosophique ancienne» (p. 31). Interroger la chaîne des sources est toujours une entreprise complexe lorsque l' auteur considéré ne cite pas lui-même l'ouvrage en question. Du côté de la tradition arabe, il serait tentant d'établir un lien entre le traité 39 de Plotin et les latins par l'intermédiaire des textes de la Plotiniana Arabica. R. Taylor a proposé un certain nombre d'arguments questionnant cette option pour ce traité même (Taylor 1998, à la p. 236 : «Textually this presents a problem for those seeking to establish strong and direct links between these passages of Arabic and Greek»). En revanche, du côté de la tradition latine, qui est celle qui nous intéresse ici, la réception du Traité 39 de Plotin est hautement étayée, notamment via Marius Victorinus, par exemple Adversus Arium I, 52, 28-30; I, 55, 19-21; III, 2, 442 (voir la notice de L. Lavaud dans Plotin, Traités 38-41, p. 195). Beierwaltes (Beierwaltes 1999) est plus positif. Le caractère fondateur de ce texte s'explique dans le contexte plus général de la réception du Timée de Platon et de son rapport au livre Lambda de la Métaphysique d'Aristote et de leur transmission dans le christianisme néoplatonicien. Ce texte de Plotin n' est cependant pas sans aspérités interprétatives.

20 Voir Armstrong 1982. Celui-ci souligne l'importance des interactions entre le monde judéo-chrétien et la philosophie grecque sur la question de la liberté créatrice: «It was not, perhaps, till the time of Plotinus, or shortly before, that, probably due to Jewish and Christian contacts, questions about God's freedom became serious and important » (Armstrong 1982, p. 399). Ce constat est précisé par Hombert 2015.

21 La liberté de l'âme réside justement dans cette tension vers le Bien «par l'intermédiaire de l'intelligence», Plotin, Ennéades, vi, 8, [39], 7 (éd. Bréhier p. 141).

22 Voir la démonstration convaincante de L. Lavaud, dans sa traduction de Plotin, en particulier p. 176-179. La position de L. Lavaud n' exclut nullement une possible reconstruction d'une objection globale par Plotin dans laquelle entreraient également des éléments du gnosticisme. 
à sa nature ${ }^{23}$; autrement dit, ce qui est absurde, c' est d' avoir le choix entre ce qui est conforme à notre nature ou contraire à notre nature. Puisqu' agir contre notre nature, c' est aller contre notre tendance volontaire au Bien, soutenir que la notre condition de notre liberté dépend de l'existence même de ce choix n' est pas raisonnable. À plus forte raison pour le Bien, serait-il absurde de soutenir que sa liberté d' agir résiderait dans le choix de faire ce qui est contraire à lui-même. Dans le cadre de l'âme, c'est la correspondance entre sa volonté et son intellection qui la rendait libre. Mais pour le Bien, il n'y a aucun acte qui suit la nature: «son être est éternellement avec son acte ${ }^{24}$, formulation très proche de celle que nous trouvons dans le Liber.

A partir de là, le traité 39 présente une ambivalence de lecture, comme si Plotin ne pouvait se résoudre à choisir entre l'hyper-transcendance du Bien et la conséquence absurde d' une absence de caractérisation du Bien libre à laquelle mène l' apophatisme ${ }^{25}$. Dans le premier cas, l'Un plotinien se distingue totalement du Dieu du Liber de causis et ouvre la voie à Proclus; dans le second, la position plotinienne s' apparente à celle du Liber. Mais dans les deux cas, une lecture simpliste du schéma émanatiste est à proscrire. Résumons brièvement ces deux positions.

1) L'Un de Plotin est au-delà de toutes choses. C'est pourquoi il faut rejeter

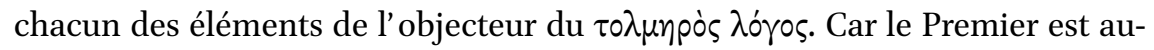

23 Plotin, Ennéades, vi, 8, [39], 7 (éd. Bréhier p. 141). Voir une excellente analyse de cette question dans O'Brien, D. 1977.

24 Plotin, Ennéades, vi, 8, [39], 7 (éd. Bréhier p. 141).

25 Dans la notice à sa traduction, E. Bréhier rappelle que « de l' aveu de tous les interprètes, ce traité est le plus «théologique» de tous ceux qu' ait écrit Plotin, c' est-à-dire celui où il est allé le plus loin pour indiquer dans quelles conditions on peut formuler des assertions sur le Premier, qui est tout à fait ineffable» (p. 119). Pour Cilento 1973, p. 114, «la méthode de persuasion » employée par Plotin ici est «une méthode de passage à la limite». O'Meara 1992 note le caractère inattendu de ce texte chez Plotin (p. 343); voir également Leroux 199o, Westra 199o et Rist 1982, p. 106. O'Meara note (O'Meara 1992, p. 348) que la voie «de persuasion» n' est pas rare chez Plotin (par ex. tr. 38 et tr. 49) et doute que ce passage du tr. 39 introduise «a new positive theology of the One» (p. 348). De Gandillac (de Gandillac 1966) avait déjà souligné l' importance de comprendre les «niveaux» lorsque l'on cherche à comprendre les perspectives parfois divergentes de Plotin (p. 199); ce que rappelle Laurent (Laurent 2011, p. 28). Indiquons simplement que cette méthode de «voie persuasive» est utilisée par Platon (par exemple Phèdre 26oa), et qu' elle n' est jamais posée comme totalement dénuée de vérité. En revanche, la réception de ce texte dans le christianisme latin n' a pas tenu compte de la question du statut du «discours persuasif» (voir Lavaud 2007, p. 197: «c'est dans la mesure même où l' intuition centrale de ce traité n'a pas été respectée, et où, plus précisément, toute la dimension métaphorique de son langage sur le premier principe a été gommée, qu'il a eu les plus grandes répercussions sur l'histoire de la pensée»). 
delà de la liberté, au-delà de l'être, et même au-delà de la nature: «Écartons donc de lui toute choses; ne disons pas même que les choses dépendent de lui et qu' il est libre; car ce sont des attributs inférieurs, qui énoncent un acte relatif à un objet différent, puisqu'ils impliquent des objets différents de l'être libre, et sur lesquels il agit sans obstacle. Or il ne doit avoir absolument aucun rapport à rien; il est ce qu'il est avant toutes choses. Supprimons même le mot: $i l$ est, et avec ce mot son rapport aux êtres. Ne disons pas non plus de lui: selon sa nature, cette expression ne s' applique qu' aux choses postérieures à lui ». L'Un est même au-delà de la nécessité ${ }^{6}$. Il est également au-delà de la volonté: «il a mis la volonté après lui » ${ }^{27}$.

Sur cette première voie, Plotin, en rejetant le schéma démiurgique d'un côté et en détachant l' Un de l'Intellect de l' autre, tout en affirmant son statut hypertranscendant ${ }^{28}$, a donc résolu la question de la liberté du Premier; mais au prix de son sacrifice ${ }^{29}$. Il a rejeté la liberté d'indifférence comme absurde et contradictoire, il a montré que l'indétermination résultant d'un choix n' était qu' un aveu d' impuissance ${ }^{30}$. Mais il a dû, de ce fait, contourner l'objection de la liberté de nature.

2) La seconde voie, qui ne trouve pas son équivalent chez Proclus et que Plotin utilise à des fins de persuasion ${ }^{31}$, contraste avec les autres traités plotiniens. Elle pose la liberté du Bien et introduit à son sujet des éléments qui devraient appartenir en propre à l'intelligence. Sur ce chemin, Plotin et le Liber présentent des proximités argumentatives troublantes, qui s' expliquent en partie par des formulations plotiniennes proches du livre Lambda de la Métaphysique

26 Plotin, Ennéades, VI, 8, [39], 9, 11-13.

27 Plotin, Ennéades, vi, 8, [39], 9, 44-47.

28 Dégager l'intellection divine de la volonté et du choix est un thème déjà présent dans le Moyen Platonisme. Voir notamment Dodds 196o; Dillon 1996; Whittaker 1978; et plus récemment, Kenney 1990.

29 Comme le dit Trouillard 1958, p. 348, avec Plotin, «la procession recule en deçà de la nature et du libre-arbitre».

30 Plotin, Ennéades, vi, 8, [39], 21, 1-7: «Dieu pouvait alors se faire autre qu'il ne s' est fait? Nullement; mais, lui enlèverons-nous son pouvoir de faire le bien, parce qu' il ne peut faire le mal? En l'Un, la puissance ne consiste pas à pouvoir les contraires; c' est une puissance inébranlable et immobile, qui est la plus grande possible, parce qu' elle ne s'écarte pas de l'Un. Pouvoir les contraires, c' est le fait d' un être incapable de se fixer dans la perfection » (trad. Bréhier).

31 Plotin, Ennéades, vI, 8, [39], 13, 1-5: «S'il faut pourtant employer ces expressions, quoiqu' elles ne soient pas correctes, disons bien encore une fois que, à parler rigoureusement, on ne doit pas admettre ici de dualité, pas même logique; mais ce que je vais dire maintenant a pour but de persuader, et il faut nous relâcher de cette rigueur dans nos formules » (trad. Bréhier). 
d'Aristote ${ }^{32}$. Pour contrer l'argument de la nécessité de nature, Plotin définit la nature du Bien comme l'unité d'un être en qui réalité et action sont identiques. Le Bien est vraiment «roi» ${ }^{33}$ parce que sa nature est d'être, d'être le Bien et d'agir non conformément à sa nature mais en étant sa nature: «il n' agit pas selon le Bien, [...] mais en tant qu'il est un, [il agit] en tant que précisément il l'est, de sorte qu'il n' est pas selon le Bien ${ }^{34}$; et plus loin: «sa nature est d'être l'être ${ }^{35}$. Dès lors, la progression de la fin du traité vise à identifier l'être et l'acte ${ }^{36}$ et à faire coïncider l'être et la volonté37, une caractéristique normalement dévolue à l' Intellect et qui constituera l' apport majeur du Liber dans sa conception de la liberté de l' acte créateur et de laquelle se départira Proclus en isolant l'action démiurgique du premier Principe. Cette unité de l'être et du faire pour désigner la liberté d' un acte volontaire, rationnel et intellectif, est conforme à l' enseignement du platonisme énoncé sous la forme du «nul n' est méchant volontairement». Afin de contrer à la fois l' absurdité d'un acte hasardeux, l'argument de la nécessité de nature de l'acte créateur et la pseudo-liberté d' indifférence, les trois écueils de la caractérisation de la liberté de l'Un-Bien, Plotin ne semble pas avoir d' autre choix que de rapprocher l'UnBien de l'Intellect.

Ainsi, en soutenant à la fois l'au-delà de la liberté de l'Un-Bien et sa liberté absolue, Plotin hésite entre deux discours sur l'Un qui ouvrent deux voies bien différentes: la première sera suivie par Proclus; la seconde par le Liber de causis. Elle définit la liberté par une adéquation de l'être et de l'acte qui est intermittente dans le cas de l'homme mais continue dans le cas de Dieu. En ouvrant la possibilité d' une telle définition, qui élimine le discours audacieux de l'accidentalité de l' essence divine, Plotin, dans son discours «persuasif», a magistralement écarté les trois objections, au prix, cependant, d'une sérieuse entorse à ses principes philosophiques.

32 Voir la notice de Lavaud 2007, p. 188.

33 On reconnait le vocabulaire du gouvernement que nous avions mentionné à propos du Liber de causis et qui rappelle la citation d'Homère par Aristote au livre Lambda de la

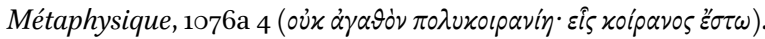

34 Plotin, Ennéades, vI, 8, [39], 9, 24, notre traduction.

35 Plotin, Ennéades, vi, 8, [39], 9, 25.

36 Plotin, Ennéades, vi, 8, [39], 12, 35-36: «il n'y a pas [...] deux choses en une, mais une seule» (trad. Bréhier).

37 Plotin, Ennéades, vi, 8, [39], 13, 29-31: «concourant à lui-même, il veut être ce qu' il est, et il est ce qu'il veut être. Sa volonté ne fait qu'un avec lui» (trad. Bréhier; nous empruntons la notion de coïncidence à la traduction de L. Lavaud, qui est une manière de rendre

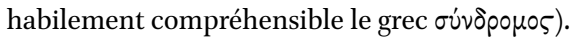



divine, enjeu de la liberté créatrice

Albert le Grand n' a pas eu directement accès à Plotin, mais il hérite, via la tradition latine et via le Liber de causis de la dimension de la volonté plotinienne que l'on a rapprochée $\S 175$ du Liber: une volonté qui n' est pas un choix entre une action conforme à la nature du Bien ou contraire à sa nature, mais une volonté réalisant, dans le champ de l'intention, l'intellection de l' acte et son assentiment. C' est à cette condition que l'émanation peut être considérée comme un acte libre et par conséquent compatible avec la notion de création.

Albert le Grand traite de la liberté et de la volonté du premier principe au livre I, traité 3 de son De causis et processu universitatis a prima causa. Le chapitre 1 de ce traité s'ouvre sur l'affirmation de la liberté inconditionnelle de l'acte du premier principe. En forçant une interprétation de livre A de la Métaphysique d'Aristote ${ }^{38}$, Albert déduit la liberté de la causalité. Le premier principe étant «causa sui » et n' ayant d'autre fin que lui-même, il est par conséquent suprêmement libre. Tout comme l' homme est souverainement libre lorsque ses actions résident en lui, a fortiori le premier principe est-il cause pour lui-même de chacune de ses actions ${ }^{39}$. Bien plus, il n' est soumis à aucune nécessité dans aucun des ordres de causalité: ni d'obligation (obligatio, cause matérielle), ni de contrainte (coactio, cause efficiente), ni d'inévitabilité (inevitabilitas, cause formelle), ni de disposition (positio, cause finale). Ainsi, pour Albert, la liberté se définit comme une totale indépendance causale: «cum igitur primus ad nullam penitus causam habeat dependentiam, constat, quod ab omni necessitate liber est ${ }^{40}$. Cinq objections assorties de cinq réponses viennent compléter cette thèse.

Comme l'auteur du Liber, Albert souligne que le Premier principe n'utilise aucun intermédiaire entre l'être et le faire dans l' acte-même du Premier. Mais que signifie «être» dans l' expression «agir par son être-même»? Le Liber ne précisait pas quel type d'être était à l' œuvre, pas plus que Plotin dans sa définition de la nature de l'Un-Bien. Albert ramène l'identité de l' être et du faire à celle de l'unité de l'esse et de l'id quod est. Agir par son être même dans le cas du Premier principe, c' est agir selon sa forme propre et non par une quelconque forme extérieure qui servirait d' instrument, comme c' est le cas dans les êtres composés. Au contraire des autres êtres qui doivent leur existence d'un

\footnotetext{
38 Aristote, Métaphysique, A, 2, 982b25-26.

39 Albert le Grand, De causis et processu universitatis a prima causa, I, tr. 3, c. 1, p. 35, l. 18-20: multo magis primus omnium dominus est, qui sibi ipsi in omni actione causa est.

Albert le Grand, De causis et processu universitatis, I, tr. 3, c. 1, p. 35, l. 46-49.
} 
autre qu' eux, l' être premier est absolument simple et se définit par l'identité de son essence et de son existence ${ }^{41}$. Cette distinction permet à Albert d' exprimer à la fois le mode de diffusion de l'émanation à partir du Premier, sa liberté et sa séparabilité d'avec les autres créatures. Dieu est totalement libre, en ce que son acte est issu de la totalité de son être mais il est également totalement séparé de sa création, puisqu' en lui seul esse et id quod est sont totalement unis. C' est véritablement parce que le premier principe est causa sui qu' il est totalement libre. Et cette démonstration est conduite à partir du Liber de causis lui-même ${ }^{42}$.

La réponse à la seconde objection est en apparence très simple: «le Premier ne peut être dit agir par nécessité à moins que son essence elle-même soit soumise à la nécessité ${ }^{43}$. Or, comme l' ont si bien montré la thèse et la réponse à la première objection, l'essence du Premier principe, pourtant nécessaire, n' est pas soumise à la nécessité parce qu' elle est cause d' elle-même.

Mais on voit bien qu'Albert ne répond que partiellement à l'objection de nature. Pour avoir une réponse plus complète, il faut se reporter à la toute fin du traité 3 , dans laquelle il exprime ce que veut dire précisément «agir par son être-même». Cette réponse est une manière plus approfondie de commenter les chapitres XIX et XXII du Liber de causis.

Nous avons vu qu' au chapitre XIX, l'auteur du Liber soulignait que le premier principe gouverne «par le mode sur lequel il agit et n' agit que par ce qu' il est (et non agit nisi per ens suum) $»^{44}$. Ce même chapitre, au $§ 158$, unit l'être, la puissance et le bien: «Le bien premier n' influe sur toutes choses ses bontés que sur un seul mode, car il n'est le bien que par son être, son étant et sa puissance (suum esse et suum ens et suam virtutem), de telle sorte qu'il est le bien, et que le bien et l'étant sont une seule chose (et bonitas et ens sunt res una) ${ }^{45}$. Si nous laissons de côté les difficultés liées à la traduction latine

41 Albert le Grand, De causis et processu universitatis, II, tr. 4, c. 3, p. 158, 1. 8-14: Quando autem agens omni simplicitate simplex est ita, quod in ipso esse et "quod est" idem est, sequitur, quod illud agens secundum totum sui a causato sui seiunctum sit et continuatorem medium non habeat, quiper esse univocum sit in utroque, sicut videmus in univoce agentibus, et quod agat sine instrumento.

42 Voir les excellentes analyses de Bonin 2001, p. 24-29.

43 Albert le Grand, De causis et processu universitatis, I, tr. 3, c. 1, p. 36, 1. 13-15: quod per essentiam agit, non potest probari, quod per necessitatem agat, nisi illa essentia subiaceat necessitati.

44 Liber de causis, XIX, 161.

45 Liber de causis XIX, 158: Et bonitas prima non influit bonitates super res omnes nisi per modum unum, quia non est bonitas nisi per suum esse et suum ens et suam virtutem, ita quod est bonitas, et bonitas et ens sunt res una. 
de l' arabe auquel Albert n' avait pas accès ${ }^{46}$, nous pouvons remarquer que le Liber emploie deux termes pour désigner l'être du Premier principe: l' esse et l'ens. Albert va reconstruire l'argument en interprétant l'ens du Liber comme un id quod est. Dans sa paraphrase, il dit en effet: « et huius causa est, quia bonitas eius, quam influit, est per suum esse et per suum ens sive 'id quod est' et per suam virtutem ita quod bonitas eius et ens sive 'id quod est', in ipsa sunt res una ${ }^{47}$. Une telle exégèse du Liber n' est pas forcée puisque l' id quod est, selon Albert ${ }^{48}$, marque précisément l' individualité de la chose. Dans les choses composées, l' absence d'identité entre l' esse et l' id quod est implique que l'être composé communique son esse à son causé tandis que son id quod est reste incommunicable ${ }^{49}$. En effet, pour qu'il y ait causalité, il faut qu'il y ait une communication d'essence qui marque la continuité entre la cause et l'effet. Une telle continuité est assurée par l'esse qui agit, à l' égard de la cause, comme un instrument ou un medium. La transmission de la forme de l'agent est la manière propre d' agir de l'être composé. N' étant pas cause de son existence et de ce qu' il est en propre, son étant, il ne peut agir que par une forme communicable qui vient s' ajouter à son être. Dans les choses composées, l' agent ne peut agir que par l'addition de «ce par quoi il agit», autrement dit, son $e s s e^{50}$. En revanche, dans le cas du Premier principe, l' agent agit de par sa nature-même, de par son ens ou encore de par son id quod est. Albert prétend ainsi montrer en quoi le Premier principe est totalement séparé de ce qu'il cause tout en lui étant le plus proche: il lui est totalement séparé et son essence reste incommunicable parce qu'il n'y a pas d'intermédiaire univoque entre l' agent et son effet; mais il lui est le plus proche parce qu'il agit de par son être-même et là encore, sans intermédiaire: «quand l' agent est absolument simple, de sorte qu' en lui être (esse) et ce qu'il est (quod est) sont identiques, il s'ensuit qu' un tel agent

46 Comme l' a bien fait remarquer Thérèse Bonin, esse traduit l' arabe anniyya et ens huwiyya que le Liber de causis traite de manière presque synonyme.

47 Albert le Grand, De causis et processu universitatis, II, tr. 4, c. 4, p. 158, 1. 58-6o, c' est nous qui soulignons.

48 Il faut être attentif à la manière proprement albertinienne d'entendre la distinction esse/id quod est.

49 Albert le Grand, De causis et processu universitatis, II, tr. 4, c. 3, p. 157, l. 74-76: « tout agent composé agit par son être et non par ce qu'il est, dans la mesure où c'est son être qui est communicable». Cette distinction repose sur le De hebdomadibus de Boèce; voir par exemple Crouse 1982.

50 Albert le Grand, De causis et processu universitatis, II, tr. 4, c. 4, p. 157, l. 87-p. 158, l. 1-2: Et hoc esse non postest nisi in his tantum, ubi est additio eius quo agitur super id quod agit, hoc est, ubi est additio esse super id quod est ( «C' est le cas seulement des choses où il y a une addition de ce par quoi on agit en plus de ce qui agit, autrement dit, lorsqu' il y a une addition de l' essence en plus du ce qui est»). 
est uni à son causé selon la totalité de son être (secundum totum sui a causato sui seiunctum sit) et qu' il ne possède aucun intermédiaire continu (continuatorem medium) qui serait univoque dans l'un et l' autre du fait de l'être (per esse univocum sit in utroque), comme c' est le cas pour les agents univoques, et qu' il agit sans instrument $»^{51}$.

Cette manière de concevoir l'action du Premier principe est fondamentale pour comprendre comment l' action du Premier est totalement libre. Comme nous le disions plus haut, la liberté de l' acte créateur réside non seulement dans la non-réciprocité de l' action, mais également dans la continuité providentielle de la relation. En agissant selon son id quod est ou son ipse ens, le premier Bien remplit ces deux conditions. Non seulement sa nature est différente des choses créées en tant qu'il est le seul à être totalement identique à lui-même dans son être, mais son être est également infiniment plus proche des choses qu'il crée que n'importe quel agent mondain ${ }^{52}$. Cette absence d'externalité continue d' un medium s' exprime non seulement dans la transmission d'une forme, comme celle du Bien ou de la sagesse par exemple, mais également dans le rapport de la nature de Dieu à son intellect ou à sa volonté.

Du côté de la transmission de la forme, Albert souligne que la production divine est similaire à ce qui se produit dans la fabrication artisanale: la forme de la maison est pensée de manière indifférente et indistincte dans l'intellect de l'artisan (forma domus in ipso intellectu artificis accipiatur, omnino indifferens et indistincta est). En revanche, elle se différencie lorsqu' elle se pense comme une structure comportant des fondations, des murs et un toit. Enfin, elle acquiert la distinction et le nombre (numerum accipit et distinctionem) lorsqu' elle se réalise concrètement dans la matière ${ }^{53}$. Cette tripartition artisanale, Albert l'emprunte clairement à Platon qui, selon Albert, a eu raison de dire que toutes choses étaient produites à partir d' un exemplaire ${ }^{54}$. L'être et le faire de la cause première se trouvent donc unies dans un intellect démiurgique platonicien qui exprime la continuité tout en rejetant l'univocité. Les formes sont unies lorsqu' elles sont en lui, différenciées dans la procession et distinctes lorsqu' elles sont engagées dans les choses constituées ${ }^{55}$.

$5^{1} \quad$ Albert le Grand, De causis et processu universitatis, II, tr. 4, c. 4, p. 158, 1. 8-14.

52 De la même manière, Thomas d'Aquin exprimera l'être dans la Somme comme ce qui est «en chaque chose ce qu'il y a de plus intime et qui pénètre au plus profond», après une analyse de la continuation et du « contact» nécessaire entre l' agent et sa production (Summa theologiae, I, 8, 1, resp. trad. 1984).

53 Albert le Grand, De causis et processu universitatis, I, tr. 3, c. 6, p. 42, l. 18-24.

54 Albert le Grand, De causis et processu universitatis, I, tr. 3, c. 6, p. 42, l. 11-13: ideo non incongrue dixit Plato, quod omnia producit sicut ab exemplo.

Albert le Grand, De causis et processu universitatis, I, tr. 3, c. 6, p. 42, 1. 6-10: Quia vero 
Du côté du rapport de l'être à la volonté, l'interprétation qu'il tire du Liber de causis, lui permet de réfuter toute position qui verrait dans le savoir ou la volonté du Premier un intermédiaire dans son acte émanatif. Ainsi Avicebron est-il dans l'erreur, lorsqu'il soutient que le Premier agit par l'intermédiaire de sa volonté ${ }^{56}$. De la même façon que Plotin soutenait que l'Un n'agissait

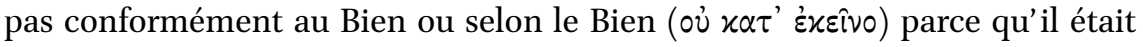
lui-même le Bien, de même Albert préserve la liberté du Premier principe en n' introduisant aucun intermédiaire entre l'être du Premier et son acte. Tant dans la transmission de la forme que dans l'unité de ce qu'il est Dieu agit donc totalement par son être. Et c' est ainsi qu'il est totalement libre.

\section{$4 \quad$ Conclusion}

S' appuyant sur le Liber de causis, Albert le Grand réconcilie donc les deux voies plotiniennes sur la question de la liberté de l'acte créateur. Tout en agissant par son être-même et tout en étant foncièrement un dans son être et dans son action, le premier principe albertinien reste au-delà de la causalité univoque et demeure séparé de ce qu'il produit. Pourtant cette séparation n'implique aucune hyper transcendance: il est véritablement la cause de ce qu' il est car c'est de son être tout entier qu'émanent toutes choses. Produisant le causé dans l'intimité de son être, l' essence du Bien premier n' est pourtant pas communicable et il agit sans se mêler aux choses ni entrer en composition avec elles. C' est tout l' enjeu du chapitre XIX du Liber de causis. La liberté du premier principe n'est donc pas celle d'un choix entre plusieurs possibles: elle se définit comme l' accomplissement ou la perfection de l'être divin sans pour autant relever d'une nécessité de nature.

Commentant le même passage du Liber de causis, la position de Thomas d'Aquin est assez proche. Il s'agit bien de montrer la proximité de la cause première tout en soulignant sa séparabilité avec le causé. Mais la solution thomasienne interprète l' intermédiaire du $§ 159$ du Liber en termes de relation, en citant notamment le $§ 122$ des Éléments de Théologie de Proclus: «tandis que les dieux rayonnent leurs biens sur ces êtres par leur être même, ou plutôt par

omnium formae triplici consideratione sunt in ipso; scilicet unitae, secundum quod in ipso sunt; et procedentes, secundum quod in lumine suo sunt, quod procedit ab ipso; et distinctae, secundum quod sunt in lumine terminato ad rei constitutionem.

56 Albert le Grand, De causis et processu universitatis, I, tr. 3, c. 3, p. 39, 1. 16-18: Avicebron autem in libro, quem Fontem vitae vocari confinxit, solus inter omnes primum principium pervoluntatem dixit. 


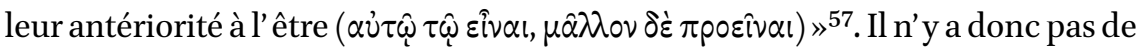
«relation $(\sigma \chi \varepsilon \dot{\varepsilon} \sigma \nu) »$ entre le dieu et son objet parce qu' il exerce son action par une «activité prénoétique» et par surabondance de sa puissance. En reprenant le terme de la «relation» mais en le transformant selon ses propres principes, Thomas interprète le Liber à la lumière de Proclus. Mais est-ce bien le sens de la version latine du Liber? Concluons en tout cas que sur la question de la liberté créatrice, Albert le Grand s' inscrit davantage dans la lignée du Liber de causis interprétée dans une dimension plus plotinienne que proclusienne, même si l'originalité d'Albert est de fonder cette liberté sur la distinction boécienne de l'esse et de l'id quod est.

Plus globalement, la liberté du Bien premier dans son acte producteur ne s'interprète jamais en termes de choix ou de libre-arbitre mais se définit en relation à l'être de la cause première et dans l'unité de celui-ci, de ses facultés et de son acte. Cette réflexion, si fondamentale pour une philosophie de la création trouve son origine chez Plotin en des termes qui interrogent déjà la conception de l'émanation nécessaire dans le néoplatonisme. Elle ouvre théologiquement la notion de création à celle d'une émanation ad extra, qui n'est pas sans lien avec la procession intra-trinitaire.

\section{Bibliographie}

\section{Sources primaires}

Albert the Great, De causis et processu universitatis a prima causa, II, 1, ed. W. Fauser, Münster, Aschendorff 1993 .

Liber de causis - édition et traduction française: P. Magnard, O. Boulnois, B. Pinchard, J.-L. Solère, La demeure de l'être. Autour d'un anonyme, Etude et traduction du Liber de Causis, Paris, Vrin, 1990.

Marius Victorinus, Adversus Arium, dans Traités théologiques sur la Trinité, éd. P. Henry, trad. P. Hadot, Paris, Cerf, 1960.

Plotin, Traités 38-41, L. Brisson et J.-F. Pradeau (dir.), Paris, G F Flammarion, 2007.

Plotin, Traité sur la liberté et la volonté de l'Un [Ennéades VI, 9 (39)], Paris, Vrin, 1990.

Plotin, Ennéades, trad. E. Bréhier, Paris, Les Belles Lettres, 1924.

Proclus. The Elements of Theology. A Revised Text with Translation, Introduction and Commentary, Oxford, Clarendon Press, 1963.

Proclus (1903) In Timaeum, éd. Diehl I, Leipzig.

Proclus (1965), Éléments de théologie, trad. J. Trouillard, Paris, Aubier Montaigne. 
Pseudo-Denys l'Aréopagite, CEuvres complètes du Pseudo-Denys l'Aréopagite, trad., comm. et notes par M. de Gandillac, Paris, Aubier, 1943.

Thomas d'Aquin, Summa theologiae, Paris, Cerf, 1984.

\section{Bibliographie secondaire}

D’Ancona, C. (1995), «La doctrine de la création mediante intelligentia», dans Ead., Recherches sur le Liber de Causis, Paris, Vrin, p. 73-95.

Armstrong, A.H. (1982), «Two views of Freedom: a Christian Objection in Plotinus, Enneads VI.8 [39] 7, 11-15?», dans E.A. Livingstone (éd.), Studia Patristica, vol. XVII in three parts, Oxford, Pergamon Press, p. 397-404.

Beierwaltes, W. (1999), «Causa sui. Plotins Begriff des Einen als Ursprug des Gedankes der Selbstursälichkeit», dans J. Cleary (éd.) Traditions of Neoplatonism. Essays in Honour of John Dillon, Aldershot / Brookfield / Singapore, Ashgate Editions, p. 191226.

Bonin, Th. (2001), Creation as Emanation. The Origin of Diversity in Albert the Great's On the Causes and the Procession of the Universe, Notre Dame (IN), University of Notre Dame Press.

Burrell, D.B. (1993), Freedom and Creation in Three Traditions, Notre Dame (IN), University of Notre Dame Press.

Burrell, D.B. (2003), «Aquinas's Appropriation of Liber de Causis to Articulate the Creator as Cause-of-Being», dans F. Kerr (éd.), Contemplating Aquinas: On the Varieties of Interpretation, London, scm Press, p. 75-83.

Cilento, V. (1973), «Libertà divina e discorso temerario », dans Saggi su Plotino, Milano, U. Mursia \& C., p. 97-122.

Crouse, R. (1982), «The doctrine of creation in Boethius. The De Hebdomadibus and the Consolatio », dans Studia Patristica 17, p. 417-421.

Dillon, J. (1996), The Middle Platonists, London, Duckworth (1977).

Dodds, E.R. (1960), «Numenius and Ammonius», dans Les Sources de Plotin, Vandoeuvres / Genève, Entretiens Fondation Hardt 5, p. 3-32.

de Gandillac, M. (1966), La sagesse de Plotin, Paris, Vrin.

Hombert, P.-M. (2015), La création chez les Pères de l'Église, Paris, Parole et Silence.

Kenney, J.P (1990), «Theism and Divine Production in Ancient Realist Theory», dans D.B. Burrell, B. McGinn (eds), God and Creation, an Ecumenical Symposium, Notre Dame (IN), University of Notre Dame Press, p. 57-8o.

Klibansky, R. (1937), The Continuity of the Platonic Tradition, London, The Warburg Institute.

Laurent, J. (2011), L'éclair dans la nuit. Plotin et la puissance du Beau, Chatou, Les Éditions de la Transparence.

Lavaud, L. (2007), «Notice», dans Plotin, Traités 38-41, L. Brisson et J.-F. Pradeau (dir.), Paris, GF Flammarion. 
Leroux, G. (1990): voir Plotin, Traité sur la liberté et la volonté de l'Un.

O’Brien, D. (1977), «Le volontaire et la nécessité. Réflexions sur la descente de l'âme dans la philosophie de Plotin », dans Revue philosophique de la France et de l'Étranger $167 / 4$, p. 401-422.

O'Meara, D.J. (1992), «The Freedom of the One», dans Phronesis 37/3, p. 343-349.

Pattin, A. (1994), «Autour du Liber de causis. Quelques réflexions sur la récente littérature», dans Freiburger Zeitschrift für Philosophie und Theologie 41, p. 354-588, (repris dans Miscellanea I. Liber de Causis, Leuven, Peeters, 2000, p. 194-228), aux pages 368370 (208-210).

Rist, J.M. (1982), Human Value. A Study in Ancient Philosophical Ethics, Leiden, E.J. Brill. Saffrey, H.-D. (1990), «Allusions antichrétiennes chez Proclus, le Diadoque Platonicien», dans Id., Recherches sur le Néoplatonisme après Plotin, Paris, Vrin, p. 201-211 (Revue des Sciences philosophiques et théologiques 59, 1975, p. 553-563).

Taylor, R. (1998), «Aquinas, the Plotiniana Arabica, and the Metaphysics of Being and Actuality», dans Journal of the History of Ideas 59, p. 217-239.

Trouillard, J. (1958), «Agir par son être même. La causalité selon Proclus», dans Revue des Sciences Religieuses 32, p. 347-357.

Westra, L. (1990), Plotinus on Freedom. A Meditation on Ennead vi.8, Lewiston (NY), E. Mellen Press.

Whittaker, J. (1978), «Numenius and Alcinous on the First Principle», dans Phoenix 32, p. 144-154. 